\title{
Updates in Treatment of Recurrent Clostridium difficile Infection
}

\author{
Tanveer Singha, Prabhjot Bedi ${ }^{\mathrm{b}, \mathrm{e}}$, Karandeep Bumrah ${ }^{\mathrm{b}}$, Jeevandeep Singh ${ }^{\mathrm{c}}$, \\ Manoj Raid ${ }^{d}$, Susmitha Seelam ${ }^{b}$
}

\begin{abstract}
Recurrent Clostridium difficile infection (CDI) is a perpetual problem that leads to increased economic burden, higher healthcare cost, and significant morbidity and mortality. Its treatment remains a challenge. While various treatment approaches have been attempted with different levels of success, robust data establishing the superiority of one approach over the others is lacking. In this article, we review the current evidence pertaining to conventional pharmacological treatment as well as fecal microbiota transplantation (FMT) as a novel, rapidly emerging treatment modality for recurrent CDI.
\end{abstract}

Keywords: Recurrent Clostridium difficile; Fecal microbiota transplantation; Tapering and pulsed vancomycin; Fidaxomicin

\section{Introduction}

Recurrence of Clostridium difficile infection (CDI) is very common leading to significant morbidity and increased healthcare costs. It is defined as a relapse of CDI symptoms within 2 8 weeks of successful treatment of the initial episode [1]. About $15-35 \%$ of CDI patients suffer from recurrent infections [2]. Frequently, CDI is a relapse of the same infection rather than a re-infection with a new strain $[3,4]$. Second and subsequent recurrences are even more common after the first recurrence [5].

\section{Risk Factors}

The literature has identified various risk factors that predispose

Manuscript submitted May 1, 2019, accepted May 31, 2019

aDepartment of Hospital Medicine, Rochester General Hospital, Rochester, NY 14621, USA

bepartment of Medicine, UPMC East, Monroeville, PA 15146, USA

'Department of Medicine, Montefiore Medical Center-Wakefield, Bronx, NY 10466, USA

dDepartment of Medicine, Michigan State University, East Lansing, MI 48824, USA

${ }^{\mathrm{e}}$ Corresponding Author: Prabhjot Bedi, Department of Medicine, UPMC East, Monroeville, PA 15146, USA. Email: prabhjotsbedi@yahoo.com

doi: https://doi.org/10.14740/jocmr3854 patients to recurrent CDI. Some of these factors are well-established, such as advanced age and antibiotic use. Controversy exists in regard to others, such as gastric acid suppression [6, 7]. Advanced age has been identified as a risk factor in multiple studies [7-9]. It has been speculated that gastric acid suppression may result in a more suitable environment for Clostridium difficile ( $C$. difficile) to transition through the stomach and proliferate; however, the data in this regard are conflicting. A small retrospective study of 125 patients and a meta-analysis of 18 observational studies found that patients receiving gastric acid-suppressing medications were at a higher risk of suffering from recurrent CDI $[8,10]$. However, a recent retrospective study that included 435 patients concluded that antisecretory medications do not increase the risk of recurrent CDI. In the same study, advanced age and partial colectomy were identified as independent risk factors [7]. Hypoalbuminemia (albu$\min <2.5 \mathrm{~g} / \mathrm{dL}$ ) was also found to be a significant risk factor in a retrospective study [8]. A recent prospective study that evaluated ATLAS score and albumin as predictors for recurrent CDI showed that only serum albumin predicted 90-day disease recurrence [11].

\section{Management Options}

\section{Tapering/Pulsed vancomycin}

The underlying rationale for tapering and/or pulsed regimens is to target the spores that are otherwise resistant to antibiotics. Once they germinate, vegetative forms are targeted by the antibiotics being given as part of a prolonged course in a tapered and/or pulsed regimen. Even though a randomized controlled trial (RCT) comparing the standard with extended duration vancomycin is lacking, current literature supports the use of extended duration vancomycin therapy. The latest Infectious Disease Society of America (IDSA) guidelines recommend tapered and pulsed vancomycin therapy for the first recurrence [1]. A recent systematic review evaluated two randomized open-label clinical trials $[12,13]$ and three case series [14-16]. Two of these case series $[14,16]$ and one of these clinical trials [13] used tapered and pulsed regimen, whereas the other case series [15] and clinical trial [12] utilized pulsed only regimen. Authors concluded that the reviewed literature supports extended duration tapering and pulsed vancomycin therapy for recurrent CDI [17]. All of these studies were small, evaluating a total of only 174 patients. Optimal treatment for recur- 
rent CDI (OpTION trial) is a randomized clinical trial that is currently recruiting. It will compare standard fidaxomicin with standard vancomycin treatment and extended duration vancomycin (standard course followed by taper and pulse) with standard vancomycin treatment alone in patients with either a first or second CDI recurrence [18] (NCT 02667418).

\section{Fidaxomicin}

Fidaxomicin (DIFICID) is a macrolide bactericidal antibiotic that is active against $C$. difficile and has been approved by the US Food and Drug Administration (FDA) for use in adults with CDI [19]. It has a narrower spectrum of activity compared to vancomycin and metronidazole, hence causes minimal disruption of the gut flora. An RCT comparing oral vancomycin and fidaxomicin that evaluated 548 patients showed similar cure rates but significantly fewer recurrence rates with fidaxomicin. However, this study only used patients with nonNAP1 (North American pulsed-field gel electrophoresis type 1) strains [20]. The NAP1 strain has a higher prevalence and reduced cure rates, and it tends to cause more severe disease $[21,22]$. But, the data pertaining to this is conflicting, as some studies have shown no association between NAP1 and severe disease [23-25]. Another RCT not only confirmed the noninferiority of fidaxomicin compared to vancomycin but also showed higher cure rates in a subgroup analysis of patients who were simultaneously receiving antibiotics for other conditions [26]. IDSA guidelines recommend a 10-day fidaxomicin course as an alternative first-line treatment for recurrent CDI [1]. A recent systematic review and meta-analysis analyzed 24 trials comparing 13 different treatment options. It concluded that amongst the available treatment options for non-multiply recurrent CDI, fidaxomicin results in a sustained symptomatic cure more frequently [27]. Another review worth mentioning is a multicenter retrospective review of CDI patients treated with fidaxomicin. Out of 97 patients who received fidaxomicin, 16 were excluded for $<8$-week follow-up. Complete response was noted in $90 \%$ and $19 \%$ developed recurrent CDI. Recurrence rate was significantly higher in patients with prior episodes of CDI ( $0 \%$ with first episode versus $23 \%$ and $29 \%$ with one and two prior episodes respectively) [28]. In a RCT of 364 patients with age $\geq 60$ years at 86 European hospitals, extended duration fidaxomicin administered in tapered and pulsed fashion was compared with a standard 10-day course of vancomycin. It revealed that extended duration fidaxomicin was superior in terms of sustained clinical cure at 30 days after the end of treatment. This benefit was seen even at 90 days follow-up. However, this trial does not specify whether the study participants were being treated for an initial or recurrent episode [29]. Fidaxomicin's premium price tag is prohibitive for its routine use. The data from studies on its overall costeffectiveness are conflicting. For instance, a study conducted in the UK showed similar costs with fidaxomicin and vancomycin in severe CDI and first recurrence, but quality-adjusted life year (QALY) gains with fidaxomicin indicated that fidaxomicin was more cost-effective. Contrarily, a study conducted in US found vancomycin to be cost-effective as compared to fidaxomicin or bezlotoxumab plus vancomycin [30].

\section{Ridinilazole}

Ridinilazole is a non-absorbable antibiotic that has proven to be successful in treating CDI in Phase 2 trials [31]. It specifically targets clostridia while causing minimal damage to the normal gut microbiota, thus minimizing collateral damage to the normal gut flora $[32,33]$. In Phase 2 trials, ridinilazole has exhibited superiority over vancomycin in sustained clinical responses [31]. Currently, a Phase 2 clinical trial comparing the safety and efficacy of ridinilazole with that of fidaxomicin is underway [34].

\section{Nitazoxanide}

Nitazoxanide, a broad-spectrum antiparasitic and antiviral drug has also been used in some cases of recurrent CDI with favorable results as evidenced by a case report from 2011 [35]. In a prospective, double-blinded study of hospitalized patients with $C$. difficile colitis, nitazoxanide was found to be just as effective as metronidazole in the treatment of $C$. difficile colitis. This study compared metronidazole for 10 days, nitazoxanide for 7 days and nitazoxanide for 10 days. There was no significant difference in the size of the symptom-free population at the end of 7 and 31 days between these groups. Even though the response rate at the end of 7 days and sustained response at 31 days was higher with nitazoxanide, the difference was not significant. Nonetheless, the sample size was not large enough to determine statistical significance [36]. In another doubleblind RCT, nitazoxanide was found to be just as effective as vancomycin in treating CDI but the sample size was very small (50) to draw any definitive conclusions [37]. There is a dearth of recent and strong quality data in this realm deterring the routine use of this antibiotic.

\section{Fecal microbiota transplantation (FMT)}

FMT, or stool transplantation, has been used with a lot of success in patients with recurrent CDI. Altered colonic microbiota, primarily due to antibiotics, is the underlying cause of recurrent $\mathrm{CDI}$ and restoration of that normal microbiota is the principle of FMT. Multiple anecdotal reports and RCTs have demonstrated the success of FMT in treating recurrent CDI. Current practice involves the use of FMT in managing second recurrences of CDI [1], but FMT may also be attempted for refractory CDI. Current evidence does not support the use of FMT as a first-line of treatment for the first recurrence. Early RCTs that compared FMT with antibiotic therapy in patients with recurrent CDI exhibited improved outcomes in patients treated with FMT compared to vancomycin $[12,38]$. For instance, in the RCT conducted by van Nood et al patients were randomly assigned to receive an initial vancomycin regimen for 4 days, followed by bowel lavage and then infusion of donor feces through a nasoduodenal tube; standard vancomycin regimen for 14 days; or standard vancomycin regimen followed just by bowel lavage. The study population had relapsed CDI after at least one previous course of adequate antibiotic 
Table 1. RCTs Comparing FMT With Pharmacotherapy

\begin{tabular}{|c|c|c|c|c|c|}
\hline Author & Sample (n) & Intervention & Comparison & $\begin{array}{l}\text { Outcome in the } \\
\text { FMT group }\end{array}$ & $\begin{array}{l}\text { Follow-up } \\
\text { period }\end{array}$ \\
\hline $\begin{array}{l}\text { Van Nood et } \\
\text { al } 2013[38]\end{array}$ & 42 & $\begin{array}{l}\text { FMT via ND tube preceded by } \\
\text { V for } 4 \text { days and bowel lavage }\end{array}$ & $\begin{array}{l}\mathrm{V} \text { for } 14 \text { days and } \mathrm{V} \text { for } 14 \text { days } \\
\text { followed by bowel lavage }\end{array}$ & $\begin{array}{l}\text { Resolution of symptoms } \\
\text { in } 15 / 16(94 \%) \text { patients }\end{array}$ & 5 weeks \\
\hline $\begin{array}{l}\text { G Cammarota } \\
\text { et al } 2015[12]\end{array}$ & 39 & $\begin{array}{l}\mathrm{V} \text { for } 3 \text { days followed } \\
\text { by FMT via C }\end{array}$ & $\begin{array}{l}\mathrm{V} \text { for } 10 \text { days followed by } \\
\text { every } 2-3 \text { days for } 3 \text { weeks }\end{array}$ & $\begin{array}{l}\text { Resolution of symptoms } \\
\text { in } 18 / 20(90 \%) \text { patients }\end{array}$ & 10 weeks \\
\hline $\begin{array}{l}\text { Susy S. Hota } \\
\text { et al } 2017[13]\end{array}$ & 30 & $\begin{array}{l}\mathrm{V} \text { for } 14 \text { days followed } \\
\text { by FMT via E }\end{array}$ & 6-week taper of V & $\begin{array}{l}\text { Resolution of symptoms } \\
\text { in } 7 / 16(43.8 \%) \text { patients }\end{array}$ & 120 days \\
\hline
\end{tabular}

RCT: randomized controlled trial; FMT: fecal microbiota transplantation; V: vancomycin; F: fidaxomicin; C: colonoscopy; E: enema; NJ: nasojejunal; ND: nasoduodenal.

treatment. In the fecal infusion group $81 \%$ patients had a resolution of diarrhea after the first infusion as compared to $31 \%$ in the vancomycin-only group and $23 \%$ in vancomycin followed by lavage ( $\mathrm{P}<0.001$ for both). The study was cut short after an interim analysis [38]. Similarly, another RCT from Italy demonstrated encouraging results of this novel therapy. Patients were randomized to receive either short 3-day regimen of vancomycin followed by one or more infusion of feces via colonoscopy, or vancomycin (125 mg four times a day for 10 days followed by 125 - $500 \mathrm{mg} /$ day every 2 - 3 days for 3 weeks at the minimum). $90 \%$ of patients in the fecal transplantation group had a resolution of diarrhea as compared to $26 \%$ in the vancomycin group $(\mathrm{P}<0.0001)$. The study was terminated after a 1-year interim analysis [12]. However, an RCT by Hota et al failed to show any significant benefits resulting from FMT. Hota et al randomized patients with recurrent CDI to receive 14 days of oral vancomycin followed by a single FMT by enema or vancomycin taper over 6 weeks [13]. This study was terminated with an interim analysis of 30 patients. In the FMT group, $43 \%$ of patients experienced resolution of their symptoms and $56 \%$ experienced recurrence of CDI. In the vancomycin group, it was $58 \%$ and $42 \%$, respectively. Contrarily a very recent single-center trial of 64 patients with recurrent CDI randomly assigned to receive FMT after 4 - 10 days of vancomycin $125 \mathrm{mg}$ four times a day, 10 days of fidaxomicin $200 \mathrm{mg}$ twice a day or vancomycin $125 \mathrm{mg}$ four times a day. In the FMT group $71 \%$ of patients achieved the primary outcome of combined clinical resolution and negative $C$. difficile toxin testing 8 weeks after the treatment; $33 \%$ and $19 \%$ of patients had the above outcome in fidaxomicin and vancomycin group respectively. Clinical resolution without a documented negative test was seen in $92 \%, 42 \%$ and $19 \%$ patients in FMT, fidaxomicin and vancomycin groups respectively [39]. The outcomes of these randomized trials are summarized in Table $1[12,13,38,39]$. A meta-analysis of seven studies by Khan et al recognized fecal transplantation as a promising modality. Their analysis found a nonsignificant trend favoring the FMT group as compared to the medical treatment group. Additionally, in a subgroup analysis, there was no significant difference between the frozen or fresh FMT in terms of resolution of diarrhea [40]. Appropriate patient selection prior to initiating
FMT is critical as a multitude of host and donor-related factors determine the success of FMT. The safety and efficacy of FMT in solid organ transplant (SOT) patients was evaluated in a retrospective, multicenter study. The authors utilized the institutional FMT databases to identify eligible patients in 10 academic centers across the United States and Canada. The primary cure was defined as complete resolution of diarrhea and/or negative $C$. difficile testing after a single FMT, and the overall cure was defined achieving those outcomes with more than one FMT with or without anti-CDI antibiotics. A total of 94 study participants received FMT for recurrent (73/94), severe (14/94) or fulminant (7/94) CDI. The primary cure was achieved in $63.8 \%(60 / 94)$ at 1-month follow-up and $58.7 \%$ (54/92) at 3-month follow-up. The overall cure rate at 3-month was at $91.3 \%(84 / 92)$ [41].

FMT can be delivered in the upper gastrointestinal (GI) tract or lower GI tract. Various modalities, including nasogastric tubes, nasoduodenal tubes, nasojejunal tube, esophagogastroduodenoscopies, or capsules (containing fecal microbiota), can be used to deliver FMT in the upper GI tract. Retention enemas or colonoscopies can be used for the lower GI tract. The benefits of one versus the other routes of administration have been compared in multiple case series, meta-analyses, and systematic reviews. Primarily, the route of instillation depends on the institutional expertise, patient preference, severity of illness, and the safest approach. Four systematic reviews and meta-analyses [42-45] showed a trend towards better results in the lower GI route compared to the upper GI route. This may have occurred because the amount of stool instillation achieved through the lower GI route was higher. A recent retrospective analysis of Israeli hospitals involving 111 patients [46] did not demonstrate any differences in the success rates of the following three study arms: upper GI route (gastroscopy, nasogastric tube, or percutaneous endoscopic gastrostomy), oral capsule, and lower GI route (colonoscopy). This study along with a systematic review by Iqbal et al [47] revealed encapsulated FMT to be promising and a safe approach for recurrent CDI provided standard protocols are followed. Further research is needed to establish optimal capsule dosing regimens and duration of treatment.

No significant adverse effects related to FMT have been 
reported. There is a risk of procedure-related complications when FMT is given through the upper or lower GI tract; however, the rate of complications should mirror the rate of complications when these procedures are performed for other reasons. Two cases of norovirus transmission have been reported in patients after receiving FMT from asymptomatic donors who did not have any sick contacts [48]. There have also been reports of inflammatory bowel disease flare-ups $[49,50]$ following FMT, with the most serious adverse effect being the necessity of a colectomy. In a retrospective analysis by Cheng et al [49] a patient who needed a colectomy had cytomegalovirus colitis following FMT, which points to the importance of donor screening prior to FMT.

In conclusion, while FMT is a safe and effective therapy for recurrent CDI, further research is necessary to establish the guidelines for FMT that include but not limited to appropriate donor screening, the timing of therapy, route of instillation, and formulation. One RCT currently being conducted in Norway is comparing metronidazole with FMT in primary CDI [51]. Future studies comparing FMT with current mainstream treatments like vancomycin and fidaxomicin would help us navigate through the treatment of recurrent CDI.

\section{Prophylaxis}

The effectiveness of oral vancomycin prophylaxis (OVP) has been examined in retrospective studies. A recently published review of three studies found a reduced risk of recurrent CDI with OVP however the data is not convincing as all of them were retrospective and lack randomization [52]. In one of these studies, OVP did not affect recurrence in patients with a single previous episode of CDI $(\mathrm{P}=0.69)$. But in patients with recurrent CDI 54.4\% in OVP group experienced a recurrence compared to $69.5 \%$ in those who did not receive OVP resulting in a number needed to treat $(\mathrm{NNT})$ of $6.6(\mathrm{P}<0.0001)$ [53]. The second reviewed study was a single-center retrospective study of 71 patients who had previous CDI and subsequently initiated on systemic antibiotics. OVP dose of 125 or $250 \mathrm{mg}$ twice daily continued along with systemic antimicrobial regimen. Recurrent CDI occurred in $4 \%$ of the vancomycin group compared to $27 \%$ in the control group with an NNT of 5 (P $<$ 0.001) [54]. Another recent retrospective single-center study from a community hospital demonstrated that administration of OVP to subjects with a history of CDI in 12 months prior to subsequent antibiotic exposure benefits in terms of risk reduction of recurrent CDI for up to 12 months [55]. The evidence currently supporting OVP is lacking in terms of long-term outcomes, adverse effects, and randomized trials. Ongoing prospective studies in this regard seek to fill this void [56-58].

\section{Discussion}

Treatment of recurrent CDI is rapidly evolving and the latest IDSA guidelines reflect that. Gupta et al in their clinical synopsis summarized the present IDSA guidelines. For the first recurrence tapered and pulsed vancomycin or fidaxomicin should be considered if the initial episode was treated with standard vancomycin. Alternatively, fidaxomicin or standard 10-day course of vancomycin can be used for those previously treated with metronidazole [59]. The evidence behind these recommendations is not strong and an ongoing RCT will likely address this [18] (NCT 02667418). Johnson et al in their letter to the editor highlighted other alternative therapies which have shown promising results in RCTs [60]. For example, in two RCTs, infusion of bezlotoxumab, a monoclonal antibody directed against toxin $\mathrm{B}$ in addition to standard antibiotic treatment was shown to reduce the rate of recurrence [61]. Ridinilazole has also shown positive results in initial studies and as additional data become available, we may see it become a part of a conventional regimen $[31,34]$. Vancomycin in a tapered and pulsed manner, vancomycin followed by rifaximin, a standard course of fidaxomicin or FMT can be used for second or subsequent recurrences per the latest IDSA guidelines. The quality of evidence for these treatment options is low except for FMT where it is moderate. The panel recommended trying appropriate antibiotics for treating at least two recurrences before resorting to FMT [1]. Treatment of recurrent CDI is a work in progress. Newly emerging antibiotics, treatment regimens and novel therapies like FMT are a testament to that.

\section{Acknowledgments}

None to declare.

\section{Financial Disclosure}

None, no external funding received.

\section{Conflict of Interest}

None to declare.

\section{Author Contributions}

All authors contributed to the review of literature, writing, and editing of the manuscript.

\section{References}

1. McDonald LC, Gerding DN, Johnson S, Bakken JS, Carroll KC, Coffin SE, Dubberke ER, et al. Clinical practice guidelines for clostridium difficile infection in adults and children: 2017 update by the Infectious Diseases Society of America (IDSA) and Society for Healthcare Epidemiology of America (SHEA). Clin Infect Dis. 2018;66(7):e1e48.

2. Marsh JW, Arora R, Schlackman JL, Shutt KA, Curry SR, Harrison LH. Association of relapse of Clostridium difficile disease with BI/NAP1/027. J Clin Microbiol. 
2012;50(12):4078-4082.

3. Figueroa I, Johnson S, Sambol SP, Goldstein EJ, Citron DM, Gerding DN. Relapse versus reinfection: recurrent Clostridium difficile infection following treatment with fidaxomicin or vancomycin. Clin Infect Dis. 2012;55(Suppl 2):S104-109.

4. Kamboj M, Khosa P, Kaltsas A, Babady NE, Son C, Sepkowitz KA. Relapse versus reinfection: surveillance of Clostridium difficile infection. Clin Infect Dis. 2011;53(10):1003-1006.

5. McFarland LV, Elmer GW, Surawicz CM. Breaking the cycle: treatment strategies for 163 cases of recurrent Clostridium difficile disease. Am J Gastroenterol. 2002;97(7):1769-1775.

6. Brown KA, Khanafer N, Daneman N, Fisman DN. Metaanalysis of antibiotics and the risk of community-associated Clostridium difficile infection. Antimicrob Agents Chemother. 2013;57(5):2326-2332.

7. Dharbhamulla N, Abdelhady A, Domadia M, Patel S, Gaughan J, Roy S. Risk Factors Associated With Recurrent Clostridium difficile Infection. J Clin Med Res. 2019;11(1):1-6.

8. Kim JW, Lee KL, Jeong JB, Kim BG, Shin S, Kim JS, Jung $\mathrm{HC}$, et al. Proton pump inhibitors as a risk factor for recurrence of Clostridium-difficile-associated diarrhea. World J Gastroenterol. 2010;16(28):3573-3577.

9. Eyre DW, Walker AS, Wyllie D, Dingle KE, Griffiths D, Finney J, O'Connor L, et al. Predictors of first recurrence of Clostridium difficile infection: implications for initial management. Clin Infect Dis. 2012;(Suppl 2):S77-87.

10. Tariq R, Singh S, Gupta A, Pardi DS, Khanna S. Association of gastric acid suppression with recurrent clostridium difficile infection: a systematic review and meta-analysis. JAMA Intern Med. 2017;177(6):784-791.

11. Knafl D, Vossen MG, Gerges C, Lobmeyr E, Karolyi M, Wagner L, Thalhammer F. Hypoalbuminemia as predictor of recurrence of Clostridium difficile infection. Wien Klin Wochenschr. 2019;131(3-4):68-74.

12. Cammarota G, Masucci L, Ianiro G, Bibbo S, Dinoi G, Costamagna G, Sanguinetti M, et al. Randomised clinical trial: faecal microbiota transplantation by colonoscopy vs. vancomycin for the treatment of recurrent Clostridium difficile infection. Aliment Pharmacol Ther. 2015;41(9):835-843.

13. Hota SS, Sales V, Tomlinson G, Salpeter MJ, McGeer A, Coburn B, Guttman DS, et al. Oral vancomycin followed by fecal transplantation versus tapering oral vancomycin treatment for recurrent clostridium difficile infection: an open-label, randomized controlled trial. Clin Infect Dis. 2017;64(3):265-271.

14. Tedesco FJ, Gordon D, Fortson WC. Approach to patients with multiple relapses of antibiotic-associated pseudomembranous colitis. Am J Gastroenterol. 1985;80(11):867-868.

15. Bakken JS. Staggered and tapered antibiotic withdrawal with administration of kefir for recurrent Clostridium difficile infection. Clin Infect Dis. 2014;59(6):858-861.

16. Sirbu BD, Soriano MM, Manzo C, Lum J, Gerding DN, Johnson S. Vancomycin taper and pulse regimen with careful follow-up for patients with recurrent clostridium difficile infection. Clin Infect Dis. 2017;65(8):1396-1399.

17. Murphy MM, Patatanian E, Gales MA. Extended duration vancomycin in recurrent Clostridium difficile infection: a systematic review. Ther Adv Infect Dis. 2018;5(6):111119.

18. Optimal Treatment for Recurrent Clostridium Difficile Full Text View - ClinicalTrials.gov [Internet]. [cited 2019 Feb 28]. Available from: https://clinicaltrials.gov/ct2/ show/NCT02667418.

19. Dificid New FDA Drug Approval | CenterWatch [Internet]. [cited 2019 Mar 18]. Available from: https://www. centerwatch.com/drug-information/fda-approved-drugs/ drug/1152/dificid-fidaxomicin.

20. Louie TJ, Miller MA, Mullane KM, Weiss K, Lentnek A, Golan Y, Gorbach S, et al. Fidaxomicin versus vancomycin for Clostridium difficile infection. N Engl J Med. 2011;364(5):422-431.

21. See I, Mu Y, Cohen J, Beldavs ZG, Winston LG, Dumyati G, Holzbauer S, et al. NAP1 strain type predicts outcomes from Clostridium difficile infection. Clin Infect Dis. 2014;58(10):1394-1400.

22. Petrella LA, Sambol SP, Cheknis A, Nagaro K, Kean Y, Sears PS, Babakhani F, et al. Decreased cure and increased recurrence rates for Clostridium difficile infection caused by the epidemic C. difficile BI strain. Clin Infect Dis. 2012;55(3):351-357.

23. Walk ST, Micic D, Jain R, Lo ES, Trivedi I, Liu EW, Almassalha LM, et al. Clostridium difficile ribotype does not predict severe infection. Clin Infect Dis. 2012;55(12):1661-1668.

24. Scardina T, Labuszewski L, Pacheco SM, Adams W, Schreckenberger P, Johnson S. Clostridium difficile infection (CDI) severity and outcome among patients infected with the NAP1/BI/027 strain in a non-epidemic setting. Infect Control Hosp Epidemiol. 2015;36(3):280-286.

25. Bauer KA, Johnston JEW, Wenzler E, Goff DA, Cook $\mathrm{CH}$, Balada-Llasat JM, Pancholi P, et al. Impact of the NAP-1 strain on disease severity, mortality, and recurrence of healthcare-associated Clostridium difficile infection. Anaerobe. 2017;48:1-6.

26. Cornely OA, Crook DW, Esposito R, Poirier A, Somero MS, Weiss K, Sears P, et al. Fidaxomicin versus vancomycin for infection with Clostridium difficile in Europe, Canada, and the USA: a double-blind, non-inferiority, randomised controlled trial. Lancet Infect Dis. 2012;12(4):281-289.

27. Beinortas T, Burr NE, Wilcox MH, Subramanian V. Comparative efficacy of treatments for Clostridium difficile infection: a systematic review and network meta-analysis. Lancet Infect Dis. 2018;18(9):1035-1044.

28. Spiceland CM, Khanna S, Pardi DS. Outcomes With Fidaxomicin Therapy in Clostridium difficile Infection. J Clin Gastroenterol. 2018;52(2):151-154.

29. Guery B, Menichetti F, Anttila VJ, Adomakoh N, Aguado JM, Bisnauthsing K, Georgopali A, et al. Extended-pulsed fidaxomicin versus vancomycin for Clostridium difficile infection in patients 60 years and older (EXTEND): a randomised, controlled, open-label, phase 3b/4 trial. Lancet 
Infect Dis. 2018;18(3):296-307.

30. Lam SW, Neuner EA, Fraser TG, Delgado D, Chalfin DB. Cost-effectiveness of three different strategies for the treatment of first recurrent Clostridium difficile infection diagnosed in a community setting. Infect Control Hosp Epidemiol. 2018;39(8):924-930.

31. Vickers RJ, Tillotson GS, Nathan R, et al. Ridinilazole for Clostridium difficile infections: safety and efficacy compared with vancomycin from the CoDIFy Phase 2 Trial. Amsterdam: Eur Congr Clin Microbiol Infect Dis. 2016.

32. Goldstein EJ, Citron DM, Tyrrell KL, Merriam CV. Comparative in vitro activities of SMT19969, a new antimicrobial agent, against Clostridium difficile and 350 gram-positive and gram-negative aerobic and anaerobic intestinal flora isolates. Antimicrob Agents Chemother. 2013;57(10):4872-4876.

33. Vickers R, Robinson N, Best E, Echols R, Tillotson G, Wilcox M. A randomised phase 1 study to investigate safety, pharmacokinetics and impact on gut microbiota following single and multiple oral doses in healthy male subjects of SMT19969, a novel agent for Clostridium difficile infections. BMC Infect Dis. 2015;15:91.

34. A study of ridinilazole (SMT19969) compared with fidaxomicin for the treatment of clostridium difficile infection (CDI) - Full Text View - ClinicalTrials.gov [Internet]. [cited 2019 Mar 5]. Available from: https://clinicaltrials. gov/ct2/show/NCT02784002.

35. Rafiullah F, Kanwal S, Majeed UM, Korsten MA, Cheema FH, Luthra M, Sohail MR. Successful use of nitazoxanide in the treatment of recurrent Clostridium difficile infection. BMJ Case Rep. 2011;2011:bcr0420114123.

36. Musher DM, Logan N, Hamill RJ, Dupont HL, Lentnek A, Gupta A, Rossignol JF. Nitazoxanide for the treatment of Clostridium difficile colitis. Clin Infect Dis. 2006;43(4):421-427.

37. Musher DM, Logan N, Bressler AM, Johnson DP, Rossignol JF. Nitazoxanide versus vancomycin in Clostridium difficile infection: a randomized, double-blind study. Clin Infect Dis. 2009;48(4):e41-46.

38. van Nood E, Vrieze A, Nieuwdorp M, Fuentes S, Zoetendal EG, de Vos WM, Visser CE, et al. Duodenal infusion of donor feces for recurrent Clostridium difficile. N Engl J Med. 2013;368(5):407-415.

39. Hvas CL, Dahl Jorgensen SM, Jorgensen SP, Storgaard M, Lemming L, Hansen MM, Erikstrup C, et al. Fecal microbiota transplantation is superior to fidaxomicin for treatment of recurrent clostridium difficile infection. Gastroenterology. 2019;156(5):1324-1332 e1323.

40. Khan MY, Dirweesh A, Khurshid T, Siddiqui WJ. Comparing fecal microbiota transplantation to standard-of-care treatment for recurrent Clostridium difficile infection: a systematic review and meta-analysis. Eur J Gastroenterol Hepatol. 2018;30(11):1309-1317.

41. Cheng YW, Phelps E, Ganapini V, Khan N, Ouyang F, Xu $\mathrm{H}$, Khanna S, et al. Fecal microbiota transplantation for the treatment of recurrent and severe Clostridium difficile infection in solid organ transplant recipients: A multicenter experience. Am J Transplant. 2019;19(2):501-511.

42. Kassam Z, Lee CH, Yuan Y, Hunt RH. Fecal microbiota transplantation for Clostridium difficile infection: systematic review and meta-analysis. Am J Gastroenterol. 2013;108(4):500-508.

43. Cammarota G, Ianiro G, Cianci R, Bibbo S, Gasbarrini A, Curro D. The involvement of gut microbiota in inflammatory bowel disease pathogenesis: potential for therapy. Pharmacol Ther. 2015;149:191-212.

44. Drekonja D, Reich J, Gezahegn S, Greer N, Shaukat A, MacDonald R, Rutks I, et al. Fecal Microbiota Transplantation for Clostridium difficile Infection: A Systematic Review. Ann Intern Med. 2015;162(9):630-638.

45. Quraishi MN, Widlak M, Bhala N, Moore D, Price M, Sharma N, Iqbal TH. Systematic review with meta-analysis: the efficacy of faecal microbiota transplantation for the treatment of recurrent and refractory Clostridium difficile infection. Aliment Pharmacol Ther. 2017;46(5):479493.

46. Greenberg SA, Youngster I, Cohen NA, Livovsky DM, Strahilevitz J, Israeli E, Melzer E, et al. Five years of fecal microbiota transplantation - an update of the Israeli experience. World J Gastroenterol. 2018;24(47):5403-5414.

47. Iqbal U, Anwar H, Karim MA. Safety and efficacy of encapsulated fecal microbiota transplantation for recurrent Clostridium difficile infection: a systematic review. Eur J Gastroenterol Hepatol. 2018;30(7):730-734.

48. Schwartz M, Gluck M, Koon S. Norovirus gastroenteritis after fecal microbiota transplantation for treatment of Clostridium difficile infection despite asymptomatic donors and lack of sick contacts. Am J Gastroenterol. 2013;108(8):1367.

49. Cheng Y, Fischer M. Fecal microbiota transplantation for recurrent or refractory Clostridium difficile infection in inflammatory bowel disease. Pract Gastroenterol. 2017;41:40-44.

50. Tran V, Phan J, Nulsen B, Huang L, Kaneshiro M, Weiss G, Ho W, et al. Severe ileocolonic crohn's disease flare associated with fecal microbiota transplantation requiring diverting ileostomy. ACG Case Rep J. 2018;5:e97.

51. Juul FE, Garborg K, Bretthauer M, Skudal H, Oines MN, Wiig H, Rose O, et al. Fecal microbiota transplantation for primary clostridium difficile infection. N Engl J Med. 2018;378(26):2535-2536.

52. Brown CC, Manis MM, Bohm NM, Curry SR. Oral vancomycin for secondary prophylaxis of clostridium difficile infection. Ann Pharmacother. 2019;53(4):396-401.

53. Carignan A, Poulin S, Martin P, Labbe AC, Valiquette L, Al-Bachari H, Montpetit LP, et al. Efficacy of secondary prophylaxis with vancomycin for preventing recurrent clostridium difficile infections. Am J Gastroenterol. 2016;111(12):1834-1840.

54. Van Hise NW, Bryant AM, Hennessey EK, Crannage AJ, Khoury JA, Manian FA. Efficacy of oral vancomycin in preventing recurrent clostridium difficile infection in patients treated with systemic antimicrobial agents. Clin Infect Dis. 2016;63(5):651-653.

55. Knight EM, Schiller DS, Fulman MK, Rastogi R. longterm efficacy of oral vancomycin prophylaxis for the prevention of clostridium difficile recurrence. J Pharm Pract. 2019. 
56. Oral vancomycin for preventing clostridium difficile recurrence - Full Text View - ClinicalTrials.gov [Internet]. [cited 2019 Mar 6]. Available from: https://clinicaltrials. gov/ct2/show/NCT03200093?term=oral\%2Bvancomyc in.

57. Efficacy of oral vancomycin prophylaxis for prevention of recurrent clostridium difficile infection - Full Text View - ClinicalTrials.gov [Internet]. [cited 2019 Mar 6]. Available from: https://clinicaltrials.gov/ct2/show/NCT0 3462459 ? cond $=$ vancomycin + prophylaxis $\& d$ raw $=4$.

58. Oral vancomycin to prevent recurrent $\mathrm{C}$ difficile infection with antibiotics - Full Text View - ClinicalTrials.gov
[Internet]. [cited 2019 Mar 6]. Available from: https:// clinicaltrials.gov/ct2/show/NCT03466502? cond $=$ oral $+\mathrm{v}$ ancomycin.

59. Gupta A, Cifu AS, Khanna S. Diagnosis and treatment of clostridium difficile infection. JAMA. 2018;320(10):1031-1032.

60. Johnson S, Gerding DN. Treatment of recurrent clostridium difficile infection. JAMA. 2019;321(5):512-513.

61. Wilcox MH, Gerding DN, Poxton IR, Kelly C, Nathan R, Birch T, Cornely OA, et al. Bezlotoxumab for prevention of recurrent clostridium difficile infection. N Engl J Med. 2017;376(4):305-317. 